\title{
BMJ Open Protocol for a scoping review on the conceptualisation of learning in undergraduate clinical nursing practice
}

\author{
Malou Stoffels, ${ }^{1,2}$ Saskia M Peerdeman, ${ }^{1,3}$ Hester E M Daelmans, ${ }^{1,4}$ \\ Johannes C F Ket, ${ }^{5}$ Rashmi A Kusurkar ${ }^{1,6}$
}

To cite: Stoffels M, Peerdeman SM, Daelmans HEM, et al. Protocol for a scoping review on the conceptualisation of learning in undergraduate clinical nursing practice. BMJ Open 2019;9:e024360. doi:10.1136/ bmjopen-2018-024360

- Prepublication history and additional material for this paper are available online. To view these files, please visit the journal online (http://dx.doi. org/10.1136/bmjopen-2018024360).

Received 23 May 2018 Revised 10 November 2018 Accepted 18 December 2018

Check for updates

(c) Author(s) (or their employer(s)) 2019. Re-use permitted under CC BY-NC. No commercial re-use. See rights and permissions. Published by BMJ.

For numbered affiliations see end of article.

Correspondence to

Malou Stoffels;

m.stoffels@vumc.nl

\section{ABSTRACT}

Introduction Learning in the clinical setting is a major form of learning in undergraduate nursing education. In spite of this, how nursing students learn in clinical practice is still largely unknown. Moreover, there is no conceptual clarity on learning in practice in the current literature. This paper aims to set up a protocol for a scoping review of the literature in order to map different conceptualisations of learning in practice in undergraduate clinical nursing education in the hospital setting. The operationalisations of different concepts will be compared and the findings of the studies will be synthesised.

Methods and analysis This scoping review will be guided by the methodological framework proposed by Arksey and 0'Malley and refined by Levac et al. and the Joanna Briggs Institute. The search strategy will be developed together with a medical information specialist and the search will be performed in electronic databases (PubMed, EBSCO/ERIC and EBSCO/CINAHL). In a first search, we will identify concepts that are used as an equivalent to learning in practice. Next, we will search for studies operationalising these concepts in undergraduate nursing education. Finally, we will check reference lists for additional publications. Abstracts and full-text studies will independently be screened by two researchers. All studies that have 'learning in undergraduate clinical nursing practice' as their main topic and that include a definition and operationalisation of an equivalent to learning in clinical practice, will be considered for inclusion. We will chart different conceptualisations and their theoretical underpinnings, as well as reported learning opportunities, informal and formal aspects of learning, social aspects of learning and gaps in the literature.

Ethics and dissemination This review will help design future studies on learning in clinical nursing practice using well-defined and agreed on terminology. The results will be disseminated through journal publications and conference presentations.

\section{INTRODUCTION}

Learning in the clinical setting is crucial for becoming a competent nurse. ${ }^{1}$ However, how nursing students learn in clinical practice is still largely unknown. A vast body of knowledge exists on factors that influence learning, but the process itself remains underexposed. ${ }^{2}$ For example, there is a lack of insight into

\section{Strengths and limitations of this study}

- This protocol outlines a rigorous design that includes an established research framework, a search strategy and a selection process.

- The search strategy includes different databases with peer-reviewed literature, with no restrictions to the study design or the publication date.

- The assessment of the quality of the included papers will enable identifying gaps in the literature.

- A limitation is that the literature search will only cover undergraduate nursing education, while a comparison with literature on learning in practice in other health professions would enrich our understanding of potential conceptualisations.

- This study will not include books or grey literature, which will allow us to map how learning in practice is conceptualised in original research.

how individual learning outcomes can be predicted, or which learning opportunities best promote students' learning. Understanding learning in the clinical setting can help design, supervise and evaluate individual learning trajectories and their outcomes in practice. This study aims to set up a protocol for a scoping review to examine how different concepts that are equivalent to 'learning in practice' are used and operationalised in the literature, and what these studies add to our understanding of learning in the clinical setting.

In the educational literature, the concept of 'learning in practice' has been widely studied in the context of workplace learning by professionals ${ }^{3}$ or practice learning by students. ${ }^{4}$ However, there is no unified definition or approach towards this concept. ${ }^{5}$ Two main characteristics of clinical learning can be clearly distinguished in the literature. First, learning in practice is often distinguished from learning in the classroom setting as informal opposed to formal learning, where informal learning arises in situations where learning is not the primary 
aim. ${ }^{6}$ However, some authors question the validity of a dichotomy between formal and informal learning, ${ }^{7}$ and state that every learning situation contains both formal and informal elements. ${ }^{8}$ This would particularly apply to clinical learning, which is characterised by a constant interplay between the 'reality' of clinical practice and formal learning interventions such as feedback and assessment. ${ }^{9}$ Another essential characteristic of workplace learning is its social rather than individual nature. ${ }^{10}$ That is, learning occurs in interaction and dialogue with others. ${ }^{6}$

In the nursing education literature, just as in other health professions education literature, different terms are used to describe and study learning in clinical practice, with different theoretical underpinnings. Moreover, the rationale behind the application of the concepts used is not always explained. Therefore, it is not always clear whether these different terms refer to the same concept, and how they are defined in terms of learning content (skills, knowledge, values), process (implicit, explicit), control (intended or unintended, guided or not guided) and learning outcomes. Also, different researchers appear to apply the same concept differently. Having clear and agreed on terminology can help design future studies that can contribute to understanding learning in clinical practice along with its limitations so that nursing wards can be organised for optimal benefit of the students.

The goal of this scoping review is to provide guidance for the use of concepts that describe learning in undergraduate clinical nursing practice in future studies. This study therefore aims to examine how different concepts that are equivalent to 'learning in practice' are used and operationalised in the literature. Therefore, we will look for studies that examine how learning in the clinical setting takes place. To enable comparison of the use of different concepts, we will focus on the general hospital setting. This context is the traditional setting for nursing training and comprises a variety of factors that may be relevant for learning, such as the presence of registered nurses, peers and other professionals, as well as complex and acute patients, thereby offering a wide array of multidimensional learning opportunities. ${ }^{11}$ We will particularly consider how formal and informal aspects of learning, as well as the social component of learning are included in these operationalisations. We will synthesise the results relating to how students learn in clinical practice.

A body of work on concepts to describe learning in practice does exist outside nursing education literature. ${ }^{12}$ To our knowledge, the only study that included distinct concepts of learning in clinical practice in a review before, was a concept analysis of work-based learning in healthcare education by Manley et al. ${ }^{13}$ The authors identified common attributes, enabling factors and consequences of workplace learning and proposed a definition. The current review will build on this work by closely examining different concepts of learning in practice in the context of undergraduate nursing education, as well as by comparing how they are used to study clinical learning. This will enable us to address gaps in the literature as well as to make suggestions for the use of terminology in future studies. Also, the current study will include literature after 2009 when Manley et $a l^{13}$ conducted their study. In interpreting our findings, we will consider the broader body of literature on learning in practice.

\section{METHODS AND ANALYSIS}

We decided to use the scoping review approach to map the different concepts that are used to study learning in clinical nursing practice as well as the way they are operationalised and the information they provide about how students learn in the clinical setting. Since the lack of a focused line of inquiry requires a broad research question, we consider a scoping review to be more appropriate than a systematic review. Scoping can help understand complex concepts through clarifying definitions and conceptual boundaries. ${ }^{14}$ Scoping will also enable us to identify key concepts, gaps in the literature, and types and sources of evidence to inform practice, policy-making and research. ${ }^{15}$ To get a comprehensive picture of the existing research, we will include studies with different designs. Since scoping reviews are hypothesis-generating rather than hypothesis-testing, this review can provide a stepping off point for further research.

Standardised reporting guidelines can help the critical appraisal of reviews and thereby increase their reproducibility, completeness and transparency. ${ }^{16}$ For systematic reviews, the Preferred Reporting Items for Systematic Review and Meta-Analysis Protocols (PRISMA-P) checklist has been developed to facilitate the preparation of a robust research protocol. ${ }^{17}$ Preferred Reporting Items for Systematic Reviews and Meta-Analyses (PRISMA) guidelines for scoping reviews are still under development. ${ }^{18}$ We therefore used relevant items of the PRISMA-P to draft this protocol, as outlined in online supplementary file 1.

To ensure rigour in reporting the methodology, we will use the six-stage approach developed by Arksey and $\mathrm{O}^{\prime}$ Malley ${ }^{19}$ and refined by Levac et $a l^{20}$ and the Joanna Briggs Institute $^{21}$ : (1) identifying the research question; (2) identifying relevant studies; (3) selecting studies; (4) charting the data; (5) collating, summarising and reporting the results; (6) expert consultation (optional and included).

\section{Stage 1: Identifying the research question}

Since our aim is to understand how learning in undergraduate clinical nursing practice is conceptualised in the current literature irrespective of research design and outcome, our research question is:

- How are different concepts that are used as an equivalent to learning in the hospital setting operationalised in the undergraduate nursing education literature?

As scoping is an iterative process, ${ }^{19}$ we might add additional questions based on our findings along the review process. While the eventual goal of this study is to contribute to the understanding of the process of nursing 
students' learning in practice, we will also synthesise results that are relevant to this topic.

\section{Stage 2: Identify relevant studies}

The search strategy will be iteratively developed by the research team. As suggested by the Joanna Briggs Institute, ${ }^{21}$ we will start with a very broad search to inform our subsequent search strategy. A comprehensive search strategy will be developed (by MS and JCFK) to conduct this stepwise search process following the Peer Review of Electronic Search Strategies 2015 guideline statement. ${ }^{22}$

In an initial search (search step 1), we will combine the terms 'learning in clinical practice' and 'undergraduate nursing students'. The search query for both steps will first be developed for PubMed and later extended to EBSCO/ERIC and EBSCO/CINAHL to identify different concepts in the literature that are used as an equivalent to 'learning in clinical practice' by nursing students. See our draft search in the online supplementary file 2 for step 1 of our search. The first 100 search results from each database will be reviewed by the researchers to assess validity of the search strategy. When agreement has been reached about the initial search strategy, the first 200 abstracts will be scanned by the two reviewers (MS and RAK) on concepts potentially eligible for inclusion in the second search step. Eligible concepts are concepts that describe the process of learning to become a nurse within the clinical context (such as 'experiential learning' or 'informal learning'), rather than specific aspects or interventions (such as 'skill acquisition' or 'peer learning'). In case of full agreement between the two reviewers on potentially eligible concepts, the first reviewer will screen the rest of the abstracts. In case of disagreement, the second author will scan another 200 abstracts until full agreement is reached. After all abstracts have been screened, the two reviewers will discuss all potentially eligible concepts and select concepts to be included in the second search step.

After having selected the different concepts, we will develop a search query (search step 2) in PubMed and subsequently extend to EBSCO/ERIC and EBSCO/ CINAHL combining each of the identified concepts with 'undergraduate nursing' to find studies operationalising one of the identified concepts in the literature on nursing students' learning in the hospital setting.

After these two searches, we will check reference lists for additional publications (see figure 1 for a flow diagram of the search and selection process). We will conduct the two searches in June 2018.

\section{Stage 3: Study selection}

Following the second step of our search strategy, two independent researchers will screen abstracts and assess the eligibility for full text retrieval. Selected full-text studies will again be compared between the reviewers with disagreement being resolved through discussion and consensus and with input from the full research team.

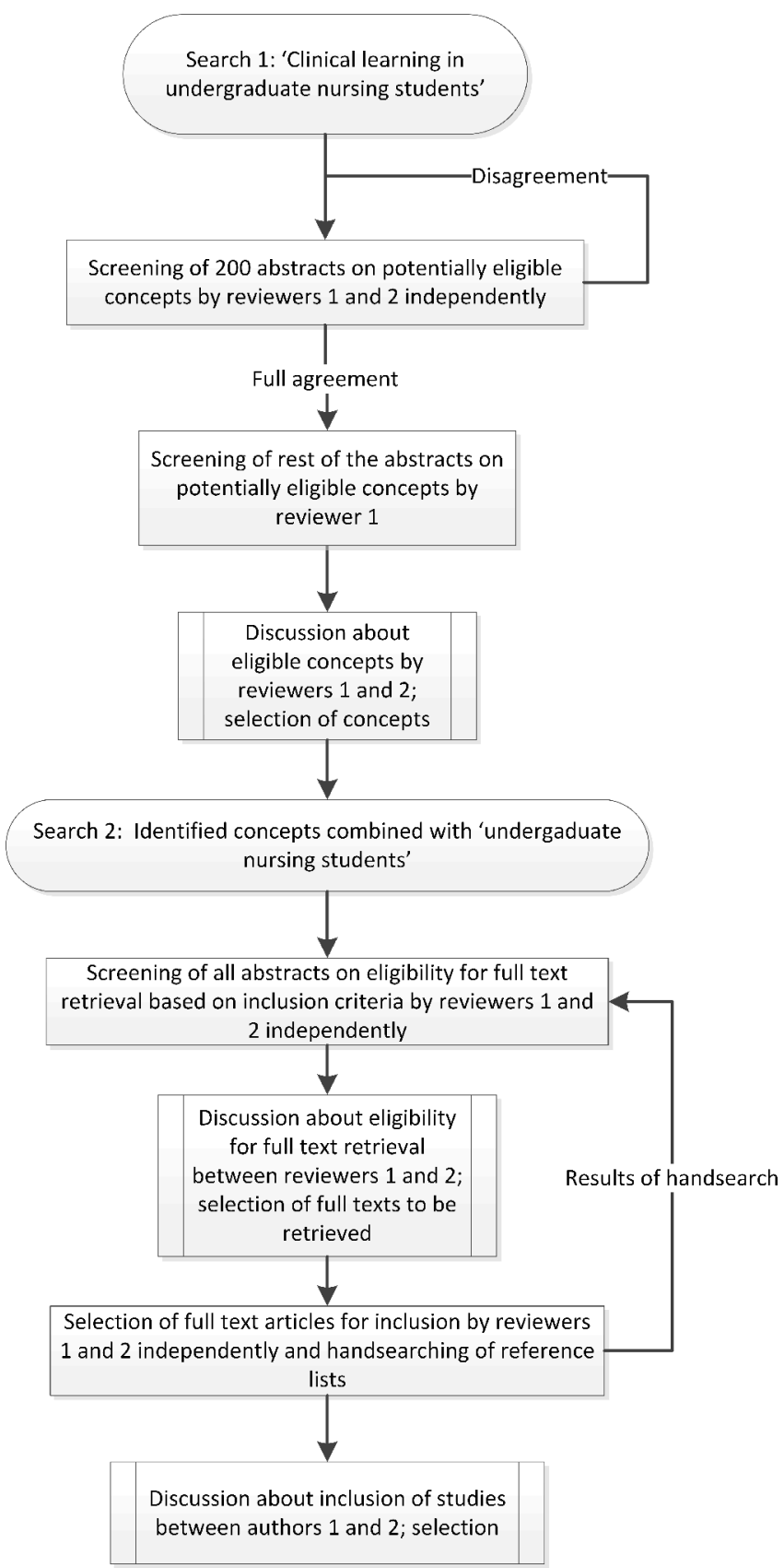

Figure 1 Flow diagram of search and study selection process.

The inclusion criteria will be developed in an iterative process in which the reviewers calibrate a threshold for inclusion and exclusion. The initial inclusion criteria will be:

- Original research or reviews in peer-reviewed journals that have learning in undergraduate clinical nursing practice in the hospital setting as one of their main topics, regardless of publication date and type of article.

- Studies that examine how students learn in the clinical hospital setting.

Since we are interested in how learning in practice is operationalised in peer-reviewed research, we exclude books, book reviews, commentaries, letters to the editor, 
$\mathrm{PhD}$ theses and reports. Reasons for exclusion will be documented at the full-text review stage.

\section{Stage 4: Charting the data}

Data will be extracted from full-text journal articles which meet the aforementioned inclusion criteria. A draft analytic frame is developed to document selected studies into an excel spreadsheet, including study characteristics (year, country, methodology, study question, study design, participants, outcomes, study quality), conceptualisation of learning in practice (definitions, theoretical underpinnings/rationale, operationalisations, formal/ informal aspects of learning, social interactions, learning opportunities) and reported gaps in the literature. Other categories that come up during the data extraction process will be discussed in the research team and added to the data extraction form. Although formal assessment of study quality is generally not performed in scoping reviews, ${ }^{21}$ some claim it should be incorporated in the methodology. ${ }^{15}$ Assessing study quality will enable us to address not only quantitative, but also qualitative gaps in the literature ${ }^{20}$ We will therefore assess the quality of included studies by a set of quality indicators for reviews developed by Buckley et $a l^{23}$ The analytic frame will be piloted on 5-10 articles by the team and will allow us to analyse the selected articles through a common framework.

We will document studies that are not selected for full text retrieval in a separate file. To ensure accurate data collection, each reviewer's independent charted data will be compared and any discrepancies will be iteratively discussed by the researchers to ensure consistency between the reviewers.

\section{Stage 5: Collating, summarising and reporting results}

A PRISMA flow diagram will be used to report final numbers in the resulting study publication. As we expect a diverse body of knowledge, we will give a descriptive account of concepts and subsequent operationalisations. We will synthesise study findings using narrative descriptions based on themes that emerge from the extracted data. The results will be compared and consolidated through consensus between two of the reviewers MS and RAK.

We will address both quantitative and qualitative gaps in the literature. We will discuss the data in the light of relevant theories on workplace learning both in and outside nursing education literature and make suggestions for the operationalisation of learning in practice for future studies.

\section{Stage 6: Expert consultation}

In order to confirm our findings and interpretations, two nurse educators with experience in scientific research and expertise on learning in clinical practice, will be approached for consultation.

\section{ETHICS AND DISSEMINATION}

This scoping review will be the first study to compare terminology used for learning in undergraduate nursing clinical practice and thereby will contribute to the design and comparison of future studies in this field. This protocol reports a comprehensive, rigorous and transparent methodology. The results will be disseminated through a peer-reviewed publication and national and international conferences such as the Association of Medical Education in Europe conference, targeting an audience involved in undergraduate healthcare education. By identifying gaps in the current body of literature, this study can guide future nursing education research. Both the methodology and the results may be of interest for researchers and educators in other health professions than nursing, given the widely spread importance of learning in clinical practice. Since the methodology applied consists of reviewing and collecting data from publicly available materials, this study does not require an ethical approval.

\section{PATIENT AND PUBLIC INVOLVEMENT}

As education is essential for improving patient care, patients will eventually benefit from the body of knowledge this study contributes to. However, specific interests of patients have not been examined. Patients have not been involved in the design nor the conduct of the study. As this concerns a review, this study has no participants.

\section{Author affiliations}

${ }^{1}$ Department of Research in Education, VUmc School of Medical Sciences, Amsterdam UMC, Amsterdam, The Netherlands

${ }^{2}$ VUmc Amstel Academy, VU University Medical Center, Amsterdam UMC, Amsterdam, The Netherlands

${ }^{3}$ Department of Neurosurgery, VU University Medical Center, Amsterdam UMC, Amsterdam, The Netherlands

${ }^{4}$ Department of Skills Training, VUmc School of Medical Sciences, Amsterdam UMC, Amsterdam, The Netherlands

${ }^{5}$ Medical Library, Vrije Universiteit, Amsterdam, The Netherlands

${ }^{6}$ LEARN! Research Institute for Learning and Education, Faculty of Psychology and

Education, VU University Amsterdam, Amsterdam, The Netherlands

Contributors MS and RAK were responsible for the initial design of this study. MS, HEMD, RAK, SMP and JCFK contributed to the development of the eligibility and data extraction criteria. JCFK and MS developed and executed the draft search strategy. MS and RAK were responsible for abstract and full-text screening. MS conceptualised the review approach and led the writing of the manuscript. MS, RAK, HEMD, SMP and JCFK contributed to and approved the final version of this protocol. RAK led the supervision of the manuscript preparation.

Funding The authors have not declared a specific grant for this research from any funding agency in the public, commercial or not-for-profit sectors.

Competing interests None declared.

Patient consent for publication Not required.

Provenance and peer review Not commissioned; externally peer reviewed.

Open access This is an open access article distributed in accordance with the Creative Commons Attribution Non Commercial (CC BY-NC 4.0) license, which permits others to distribute, remix, adapt, build upon this work non-commercially, and license their derivative works on different terms, provided the original work is properly cited, appropriate credit is given, any changes made indicated, and the use is non-commercial. See: http://creativecommons.org/licenses/by-nc/4.0/. 


\section{REFERENCES}

1. Cope P, Cuthbertson P, Stoddart B. Situated learning in the practice placement. J Adv Nurs 2000;31:850-6.

2. Flott $E A$, Linden $L$. The clinical learning environment in nursing education: a concept analysis. J Adv Nurs 2016;72:501-13.

3. Billett S. Learning through work: workplace affordances and individual engagement. J Workplace Learn 2001;13:209-14.

4. Guile D, Griffiths T. Learning Through Work Experience. J Educ Work 2001;14:113-31.

5. Manuti A, Pastore S, Scardigno AF, et al. Formal and informal learning in the workplace: a research review. Int J Train Dev 2015;19:1-17.

6. Tynjälä P. Perspectives into learning at the workplace. Educ Res Rev 2008:3:130-54.

7. Billett S. Critiquing workplace learning discourses: Participation and continuity at work. Studies in the Education of Adults 2002;34:56-67.

8. Janssens L, Smet K, Onghena P, et al. The relationship between learning conditions in the workplace and informal learning outcomes: a study among police inspectors. Int J Train Dev 2017;21:92-112.

9. Andrews GJ, Brodie DA, Andrews JP, et al. Professional roles and communications in clinical placements: a qualitative study of nursing students' perceptions and some models for practice. Int J Nurs Stud 2006;43:861-74.

10. Eraut M. Informal learning in the workplace. Studies in Continuing Education 2004;26:247-73.

11. Bisholt B, Ohlsson U, Engström AK, et al. Nursing students assessment of the learning environment in different clinical settings. Nurse Educ Pract 2014;14:304-10.

12. Billett S. Mimesis: learning through everyday activities and interactions at work. Hum Resource Dev Rev 2014;13:462-82.

13. Manley K, Titchen A, Hardy S. Work-based learning in the context of contemporary health care education and practice: a concept analysis. Pract Dev Health Care 2009;8:87-127.
14. Davis K, Drey N, Gould D. What are scoping studies? A review of the nursing literature. Int J Nurs Stud 2009;46:1386-400.

15. Daudt HM, van Mossel C, Scott SJ. Enhancing the scoping study methodology: a large, inter-professional team's experience with Arksey and O'Malley's framework. BMC Med Res Methodol 2013;13:48.

16. Colquhoun HL, Levac D, O'Brien KK, et al. Scoping reviews: time for clarity in definition, methods, and reporting. $J$ Clin Epidemiol 2014:67:1291-4.

17. Moher $\mathrm{D}$, Shamseer L, Clarke M, et al. Preferred reporting items for systematic review and meta-analysis protocols (PRISMA-P) 2015 statement. Syst Rev 2015;4:1.

18. Tricco A, Straus S, Moher D. Preferred reporting items for systematic reviews and meta-analysis: extension for Scoping Reviews (PRISMAScR). 2017. EQUATOR Netw http://www equator-network org/ wp-content/uploads/2009/02/Executive-summary_ScR_Dec-9 pdf (accessed 20 May 2017).

19. Arksey H, O'Malley L. Scoping studies: towards a methodological framework. Int J Soc Res Methodol 2005;8:19-32.

20. Levac D, Colquhoun H, O'Brien KK. Scoping studies: advancing the methodology. Implement Sci 2010;5:69.

21. Peters M, Godfrey C, Mclnerney P, et al. Chapter 11: Scoping Reviews. In: A E, M Z, eds. Joanna Briggs Institute Reviewer's Manual: The Joanna Briggs Institute, 2017.

22. McGowan J, Sampson M, Salzwedel DM, et al. PRESS Peer Review of Electronic Search Strategies: 2015 Guideline Statement. J Clin Epidemiol 2016;75:40-6.

23. Buckley S, Coleman J, Davison I, et al. The educational effects of portfolios on undergraduate student learning: a Best Evidence Medical Education (BEME) systematic review. BEME Guide No. 11. Med Teach 2009;31:282-98. 